\title{
Namenregister zu Band 55
}

Die íettgedruckten Zahlen bedeuten Eigenarbeiten.

A.

Abderhalden 131. Abelsdorf 343. Albrich 98. Alexander 64. Altland 259, 260. Arnold 129, Asmus 104.

Aszalós 119.

Aust 427.

A xenfeld 781,

B. Bar 262, 393.

Barbieri 427. Barkan 82.

$>, 2$

Bartels 73, 240, 244, 257,

259, 262. Baurmann 429. Becker 75. Beckershaus 181. Behr 74, 81, 82, 293. Berner 426.

Bertolotti 109.

Nanienregister.

441

Beselin 105. Besso 278, 285. Bielschowsky 81, 250,

251. v. Blakovics $\beta 1,115,116$,

$117,119,120,361,362$,

363. Blatt 69. Blegvad 73. Block 350. Boegehold 61. Bögel 259. Böhm 77, 107. Borries 243, 246, 247. Brana 117, 120, 361. Braunschweig 85. Braunstein 254, 364. Brooser 115. Bruckner 86. Burger 80, 326. Buschke 344.

C.

Candian 68. Cemach 244. Charlin 101. Clausen 292 (P.). Colden 426.

Comberg69, 80, 203, 343. Cords 238, 320. Csapody 98, 117, 118, 120, 121, 362, 364. Cusumano 428.

D.

Deutsch 349, 351. Dim itz 64. Dimmer 354. Dinger 66, 73. Ditroi 112, 119. Dohme 88, 344.

Domarus 81. Dusser de Barenne 248.

E. Edeskuty 38. Egtermeyer 367. Eisner 208. Eliasberg 105. Elschnig 66, 67, 103. Engelking 240. Enroth 66, 67. Enslin 345. Erben 426. Erdmann 260, 430. Erggelet 69, 70. Esser 82.

F.

Fazakas 115, 118. Fehr 203.

Feigenbaum 430.

Feilchenfeld 100.

Fischel 357.

Fischer, F. 190, 242, 272.

Flaschenträger 345.

Fhiri 27. 
Framin 365 .

Fremel 248.

Frey 72.

Fricke 367.

Friede 70, 71, 220, 429.

v. Friedrich 368.

Fuchs, A. 79, 195, 272,

275. Fuchs, E, 75, 76, 191,

195, 427. G. Gallati 60. Gallus 67, 256. Gatscher 245. Genna 429. Geyer 78. Gifford 427. Gilbert

77. Gleichen 60, 86, 87. Greeff 78, 85. Griesmann 245. Grimminger 144, 235. Grönholm 71, 78.

v. Grósz 116, 118, 120,

362. Grüter 279. Guggenheim 161. Guist 198, 209, 269, 271,

308, 318. Gutzeit 366.

$\mathrm{H}$.

Hagen 67. Haist 81. Halász 361. Hamburger 108. Handmann 107, 254. Hanssen 73, 75, 76.

Hartinger 86. Heine 1, 102. Henker 87. Hensen 78, 104. Heßberg 51, 258, 259,

260, 261. Hubert 81. Hildesheimer 345. Hirschberg, J. 289 (P),

354 (P.). Hockenbichler, 428. v. Hoesslin 131. van der Hoeve 213. 365,

368. Hoffmann, F. B. 343. Hoffmann, W. 164, 245. Holth 426.

Horay 115. Horn 367.

v. Horváth 119, 363 Huber 114, 117, 120, 361, 363.

i.

Igersheimer 85.

Imre jun. 113, 115, 117,

118. Incze $114,121,361,363$.

Invuye 77. Isakowitz 64.

J.

Jablonski 65. Jacobv, J. 427. Jahn 85. Janker 429. Jeß 81, 98. de Jongh 284. Junius 74.

$\mathrm{K}$.

Kafka 191. Karbowski 248. Karpow 82, 129. Kayser 71. Kestenbaum 70, 80, 238,

244, 267, 274. Kestermann 81. Kirsch 61. Klainguti 217. de Klejn 246, 248. Kobrak 246. Kolb

80. Koch 257.

Köllner 239, 244, 245. Korff-Petersen 62. Kramer, R. 16, 79, 84,

88, 113, 353. Kraupa 100, 101, 253. Kreidl 245. Kreiker 45, 113, 114, 117,

119. Kronfeld 192, 193. Kropp 258. Kubik 99, 253, 428. Kühl 83, 86, 87. Kümmell 281. Kuschel 401.

ii)

Landwehr 68.

Lang 278.

Laqueur 206.

Lauber 62, 105, 188, 292

(P.), 353. Leidler 248. Levins ohn 73, 74, 81,

203. Licsko 99.

442 Namenregister.

v. Liebermann 114, 362,

363. Lindner 79, 349, 353. Löhlein 61, 82. Lohmann 82. Löwenstein 82, 84, 103, 
131. Löẁy 249. Lundsgaard 66, 67. Lutz 240, 252.

M.

Magnus 246. Marquez 79, 130, 252. Martens 206. di Marzio 112. Maschino 428. Mauksch 189, 196, 276. Mayer 248. Mehner 105. Meisling 243. Meisner 99. Meißner 269, 428. Mennerich 255. Meyer 431 v. Mó cs y 118. Monjukawa 426. Moore 122. Mügge 64.

Müller, F. 86, 88, 131. Müller, P. 192. Munch 69. Mylius 133.

N. Nasr Farid Bey 80. Neuhann 259, 262. Neuhäuser 97. No nay 118.

0 .

Ohm 240, 241, 242, 244. Oláh 115. Oppenheimer 85.

P. Paderstein 1.39, 345. Pándy 363. Pásztor 116. Pelláthy 364. Péterfi 118. Peters 428. Petres 116, 363. Pichler 85. Pillat267, 268, 352.

Pincus 77, 253. Planner 272. Plocher 76. Podesta 431. Pollock 427. Pöllot 66, 67. Purtocher 80. ß.

Raband 427.

Raeder 59, 83, 95.

Raskop 86.

Raum 78.

Reganate 429.

Reis 248.

Reitsch 107.

Remak 67.

Richter 61.

Riedt 72, 429.

Rieger 346.

v. Rohr 85, 87.

Rohrschneider 346.

Rönne 79, 83.

Röper 81 .

Rosen 84.

Roseníeld 248.

Rosenhauch 68.

Rosenstein 67.

Rotfeld 248.

Rotter 421.

Rotth 113, 114, 118, 120,

121. Ruttin 247.

S. Sachs 366. Safar 101, 195, 428. Sallmann 93, 200, 250. Sal us 68, 100, 108. Salzmann 58. Samojloff 97. Sattler 102. Schall 207. Scheerer 102. Schiötz 67, 128. Schmidt, H. 373. Scho ttenheimer 85. Schrader 67. Schubeus 255. Schulek 113. Schwarz 61. Seefelder 424. Seligmann 345. i Siegrist 432.

Silívast 430.

Singer $117,118,362$.

Sommer 263, 352.

Sorgo 204.

Spezialeli vincione429.

Stargardt 207. 
Ste vens 429 .

Stoewer 344.

Screbel 62, 104.

Screisch 64, 80.

Szarvassy 116.

v. Szé k el y 117.

v. Szokolik 117, 118.

$\mathrm{T}$.

Tauß ig 266. Thiel 96. Thieß 129. Tobias 187 . Torrigiani Ill.

U.

Uhthoíf 74. Urbahn 262. Urbanek 195.

V. Versteegh 246. Vogt 60, 72.

W.

Weckert 85. Wegner 381. Weintraub 272. Weiß 78, 85, 86, 288. Wendt 256. W e r n i c k e 73.

Werbitzky 58. Wick 322, 432. Wiener 206. Wiesenthal 203. Wilbrand 292 (P.), 371. Wilmsen 259. Wiltschke 107. Wißmann 123. Wolff 84, 284. Wolffberg 61.

Z.

Zahn .

72

429. Zaun 242. Zeemann 280. Zilles 225

324. iZimmermann 81

283. 